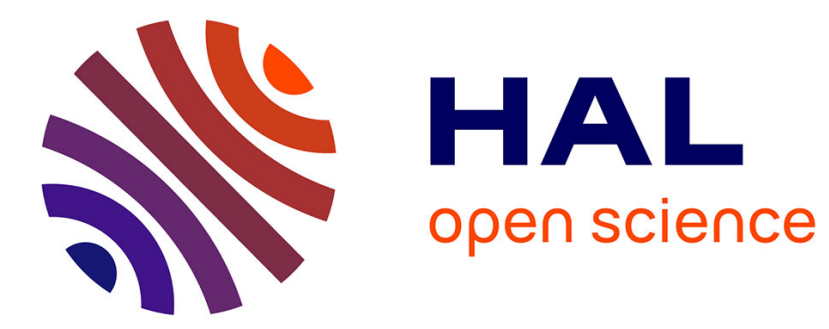

\title{
RATE REGION OF CORRELATED MIMO MULTIPLE ACCESS CHANNELS AND BROADCAST CHANNELS
}

Romain Couillet, Merouane Debbah, Jack W. Silverstein

\section{- To cite this version:}

Romain Couillet, Merouane Debbah, Jack W. Silverstein. RATE REGION OF CORRELATED MIMO MULTIPLE ACCESS CHANNELS AND BROADCAST CHANNELS. IEEE Workshop on Statistical Signal Processing, Aug 2009, United Kingdom. 4 p. hal-00447034

\section{HAL Id: hal-00447034}

https://hal-centralesupelec.archives-ouvertes.fr/hal-00447034

Submitted on 14 Jan 2010

HAL is a multi-disciplinary open access archive for the deposit and dissemination of scientific research documents, whether they are published or not. The documents may come from teaching and research institutions in France or abroad, or from public or private research centers.
L'archive ouverte pluridisciplinaire HAL, est destinée au dépôt et à la diffusion de documents scientifiques de niveau recherche, publiés ou non, émanant des établissements d'enseignement et de recherche français ou étrangers, des laboratoires publics ou privés. 


\section{RATE REGION OF CORRELATED MIMO MULTIPLE ACCESS CHANNELS AND BROADCAST CHANNELS}

Romain Couillet, Mérouane Debbah

\author{
ST-Ericsson, Supélec \\ Plateau de Moulon, 3 rue Joliot-Curie \\ 91192 Gif sur Yvette, France \\ \{romain.couillet,merouane.debbah\}@supelec.fr
}

\author{
Jack W. Silverstein* \\ Department of Mathematics \\ North Carolina State University \\ Raleigh, North Carolina 27695-8205 \\ jack@math.ncsu.edu
}

\begin{abstract}
In this paper, the rate region of large multi-antenna multiple access channels and broadcast channels are investigated. The propagation channels between transmitters and receivers are modelled as independent Gaussian with separable variance profiles. It is shown in particular that the large antenna rate regions do not depend on the specific channel realization, but only on the channel transmit and receive covariance matrices. The theoretical results are corroborated by simulations.
\end{abstract}

\section{INTRODUCTION}

When multiple-input multiple-output (MIMO) theory arose, the problem of transmission rate limitations seemed to have been solved as large capacity gains were predicted [1]. However, recent applications of MIMO technologies prove more challenging, since the promised rate increase only arises for high signal-to-noise ratio (SNR) and low antenna correlation. Moreover, it is still difficult understand the different effects of antenna correlation on the channel capacity of a multiuser MIMO system for channels other than parallel Gaussian or uncorrelated Rayleigh. Among the main contributions to the study of the effects of correlation to wireless communications, [2] derives the capacity of a single-user MIMO system in Rayleigh fading with general variance profile, when both numbers of transmit and receive antennas grow large. The extension of this work to single-user decoding in multi-cell network with interference and separable channel variance profiles was explored in the case of large numbers of antennas in [3], by applying the mathematically involved replica method. In [5], the same problem is addressed using analytical random matrix theory tools and especially Stieltjes transforms.

In the following, we derive the complete achievable rate region of multi-user wireless communications, when the propagation channels are modelled as independent Gaussian matrices with separable variance profiles ${ }^{1}$. We shall therefore

\footnotetext{
* Silverstein's work is supported by the U.S. Army Research Office under Grant W911NF-05-1-0244.

${ }^{1}$ this channel model is also known as the Kronecker model.
}

successively provide an expression for the rate region of multiple access channels (MAC) and their dual broadcast channels (BC), when either the transmitter performs uniform power allocation, or when the eigenvectors of the transmit covariance matrix are aligned to those of the transmit antenna correlation matrix and the eigenvalues of the transmit covariance matrix are obtained by convex optimization. This solution is known to be optimal if there exist no correlation at the reception [4]. Additionally, we assume a large number of antennas both at the transmit and at the receive sides. To derive the $\mathrm{BC}$ rate region, we will use a new fundamental result of random matrix theory, which provides a deterministic equivalent to the Stieltjes transform of a certain type of matrices [5].

The rest of this paper unfolds as follows: in Section 2, we introduce random matrix theory results needed in the following. In Section 3, we present the uplink MAC and downlink $\mathrm{BC}$ channel models. In Section 4, we derive the rate region of the MAC channel which, by duality, leads to solve the rate region of the $\mathrm{BC}$ channel. In Section 5, we provide simulation results of the previously derived formulas. Finally, we provide our conclusions in Section 6.

Notation: In the following, boldface lower-case symbols represent vectors, capital boldface characters denote matrices ( $\mathbf{I}_{N}$ is the $N \times N$ identity matrix). $X_{i j}$ denotes the $(i, j)$ entry of $\mathbf{X}$. The Hermitian transpose is denoted $(\cdot)^{\mathrm{H}}$. The operators tr $\mathbf{X},|\mathbf{X}|$ and $\|\mathbf{X}\|$ represent the trace, determinant and spectral norm of matrix $\mathbf{X}$, respectively. The symbol $\mathrm{E}[\cdot]$ denotes expectation. The notation $F^{\mathbf{Y}}$ stands for the empirical distribution of the eigenvalues of the Hermitian matrix $\mathbf{Y}$.

\section{MATHEMATICAL PRELIMINARIES}

Theorem 1 Let $K, N \in \mathbb{N}$ be some positive integers. Let

$$
\mathbf{B}_{N}=\sum_{k=1}^{K} \mathbf{T}_{k}^{\frac{1}{2}} \mathbf{X}_{k}^{\mathrm{H}} \mathbf{R}_{k} \mathbf{X}_{k} \mathbf{T}_{k}^{\frac{1}{2}}
$$

be an $N \times N$ matrix, such that, for all $k \in\{1, \ldots, K\}, \mathbf{R}_{k}$ is $n_{k} \times n_{k}$ Hermitian nonnegative, $n_{k} \in \mathbb{N} ; \mathbf{T}_{k}^{\frac{1}{2}}$ is the $N \times N$ 
Hermitian nonnegative definite square root of some nonnegative $\mathbf{T}_{k}$; the sequences $\left\{F^{\mathbf{R}_{k}}\right\}_{n_{k} \geq 1}$ and $\left\{F^{\mathbf{T}_{k}}\right\}_{N \geq 1}$ are tight, i.e. for all $\varepsilon>0$, there exists $M_{0}>0$ such that $M>M_{0}$ implies $F^{\mathbf{R}_{k}}([M, \infty))<\varepsilon$ and $F^{\mathbf{T}_{k}}([M, \infty))<\varepsilon$ for all $n_{k}, N ; \mathbf{X}_{k}$ is $n_{k} \times N$ with i.i.d. complex Gaussian entries of variance $1 / n_{k}$.

For $k \in\{1, \ldots, K\}$, let $c_{k}=n_{k} / N$. Also denote, for $z \in \mathbb{C} \backslash \mathbb{R}^{+}, m_{N}(z)=\frac{1}{N}\left(\mathbf{B}_{N}-z \mathbf{I}_{N}\right)^{-1}$. Then, as all $n_{k}$ and $N$ grow large (while $K$ is fixed), with ratio $c_{k}$

$$
m_{N}(z)-m_{N}^{o}(z) \stackrel{\text { a.s. }}{\longrightarrow} 0
$$

where

$$
m_{N}^{o}(z)=\frac{1}{N} \operatorname{tr}\left(\sum_{k=1}^{K} \int \frac{r_{k} d F^{\mathbf{R}_{k}}\left(r_{k}\right)}{1+\frac{r_{k}}{c_{k}} e_{k}(z)} \mathbf{T}_{k}-z \mathbf{I}_{N}\right)^{-1}
$$

and the set of functions $\left\{e_{i}(z)\right\}, i \in\{1, \ldots, K\}$, form the unique solution to the $K$ equations

$$
e_{i}(z)=\frac{1}{N} \operatorname{tr} \mathbf{T}_{i}\left(\sum_{k=1}^{K} \int \frac{r_{k} d F^{\mathbf{R}_{k}}\left(r_{k}\right)}{1+\frac{r_{k}}{c_{k}} e_{k}(z)} \mathbf{T}_{k}-z \mathbf{I}_{N}\right)^{-1}
$$

such that $\operatorname{sgn}\left(\Im\left[e_{i}(z)\right]\right)=\operatorname{sgn}(\Im[z])$.

The function $m_{N}(z)$ is the Stieltjes transform [9] of the random variable with cumulative distribution function $F^{\mathbf{B}_{N}}$.

Theorem 2 Let $\mathbf{B}_{N}$ be a random Hermitian matrix as defined in Theorem 1 with the additional assumption that there exists $M>0$, such that, for all $N, n_{k}, \max \left(\left\|\mathbf{T}_{k}\right\|,\left\|\mathbf{R}_{k}\right\|\right)<$ $M$, and let $x>0$. Then, for large $N, n_{k}, \mathcal{V}(x)-\mathcal{V}^{\circ}(x) \stackrel{\text { a.s. }}{\longrightarrow}$ 0 , where

$$
\begin{aligned}
\mathcal{V}(x) & =\log \left|\mathbf{I}+\frac{1}{x} \mathbf{B}_{N}\right| \\
\mathcal{V}^{o}(x) & =\int_{x}^{+\infty}\left(\frac{1}{w}-m_{N}^{(0)}(-w)\right) d w
\end{aligned}
$$

Both theorems above are thoroughly proven in [5].

\section{SYSTEM MODEL}

Consider a wireless multi-user channel with $K \geq 1$ users indexed from 1 to $K$, controlled by a single base-station. User $k$ is equipped with $n_{k}$ antennas while the base-station is equipped with $N$ antennas. We additionally denote $c_{k}=n_{k} / N$.

Even if we will mainly focus on the uplink MAC channel between the $K$ users and the base-station, our objective is to characterize the downlink $\mathrm{BC}$ channel between the basestation and the users. For this, we denote $\mathbf{s} \in \mathbb{C}^{N}, \mathrm{E}\left[\mathbf{s s}^{\mathrm{H}}\right]=$ $\mathbf{P}$, the signal transmitted by the base-station, with power constraint $\operatorname{tr}(\mathbf{P}) \leq P$, for some sum-power $P>0 ; \mathbf{y}_{k} \in \mathbb{C}^{n_{k}}$ denotes the signal received by user $k$ and $\mathbf{n}_{k} \sim \operatorname{C\mathcal {N}}\left(0, \sigma^{2} \mathbf{I}_{n_{k}}\right)$ the noise vector received by user $k$. The fading MIMO channel between the base-station and user $k$ is denoted $\mathbf{H}_{k} \in$ $\mathbb{C}^{N \times n_{k}}$. Moreover, we assume that $\mathbf{H}_{k}$ has a separable variance profile, i.e. can be decomposed as

$$
\mathbf{H}_{k}=\mathbf{R}_{k}^{\frac{1}{2}} \mathbf{X}_{k} \mathbf{T}_{k}^{\frac{1}{2}}
$$

with $\mathbf{R}_{k} \in \mathbb{C}^{n_{k} \times n_{k}}$ the Hermitian correlation matrix at receiver $k$ with respect to the channel $\mathbf{H}_{k}, \mathbf{T}_{k} \in \mathbb{C}^{N \times N}$ the correlation matrix at the base-station for the $\mathbf{H}_{k}$ link and $\mathbf{X}_{k} \in$ $\mathbb{C}^{n_{k} \times N}$ a random matrix with Gaussian independent entries of variance $1 / n_{k}$.

With the assumptions above, the downlink communication model unfolds

$$
\mathbf{y}_{k}=\mathbf{H}_{k} \mathbf{s}+\mathbf{n}
$$

Denoting equivalently $\mathbf{s}_{k}$ the signal transmitted in the dual uplink by user $k$, such that $\mathrm{E}\left[\mathbf{s}_{k} \mathbf{s}_{k}^{\mathrm{H}}\right]=\mathbf{P}_{k}, \operatorname{tr}\left(\mathbf{P}_{k}\right) \leq P_{k}$, for some transmit power $P_{k} \geq 0, \mathbf{y}$ and $\mathbf{n}$ the signal and noise received by the base-station, we have the dual uplink model

$$
\mathbf{y}=\sum_{k=1}^{K} \mathbf{H}_{k}^{\mathrm{H}} \mathbf{s}_{k}+\mathbf{n}_{k}
$$

In the following, we will derive the $\mathrm{BC}$ rate region by means of the MAC-BC duality [7]. We consider first the MAC capacity region.

\section{CAPACITY REGION}

\subsection{Multiple Access Channels}

The capacity $\mathrm{C}_{\mathrm{MAC}}\left(P_{1}, \ldots, P_{K} ; \mathbf{H}^{\mathrm{H}}\right)$ of the MAC channel $\mathbf{H}^{\mathrm{H}}$ under respective transmit power constraints $P_{1}, \ldots, P_{K}$ for users $1, \ldots, K$ respectively and compound channel $\mathbf{H}^{\mathrm{H}}=$ $\left[\mathbf{H}_{1}^{\mathrm{H}} \ldots \mathbf{H}_{K}^{\mathrm{H}}\right]$, is given in [6], and reads

$$
\begin{aligned}
& \mathcal{C}_{\text {MAC }}\left(P_{1}, \ldots, P_{K} ; \mathbf{H}^{\mathrm{H}}\right) \\
& =\bigcup_{\substack{\operatorname{tr}\left(\mathbf{P}_{i}\right) \leq P_{i} \\
\mathbf{P}_{i} \leq 0 \\
i=1, \ldots, K}}\left\{\left\{R_{i}, 1 \leq i \leq K\right\}: \sum_{i \in \mathcal{S}} R_{i}\right. \\
& \left.\quad \leq \log \left|\mathbf{I}+\frac{1}{\sigma^{2}} \sum_{i \in \mathcal{S}} \mathbf{H}_{i}^{\mathrm{H}} \mathbf{P}_{i} \mathbf{H}_{i}\right|, \forall \mathcal{S} \subset\{1, \ldots, K\}\right\}
\end{aligned}
$$

For any set $\mathcal{S} \subset\{1, \ldots, K\}$, from 1 and 2, we have approximately, for $N, n_{k}$ large,

$$
\log \left|\mathbf{I}+\frac{1}{\sigma^{2}} \sum_{i \in \mathcal{S}} \mathbf{H}_{i}^{\mathrm{H}} \mathbf{P}_{i} \mathbf{H}_{i}\right|=\int_{\sigma^{2}}^{\infty}\left(\frac{1}{w}-m_{\mathcal{S}}^{o}(-w)\right)
$$

where

$$
m_{\mathcal{S}}^{o}(z)=\frac{1}{N} \operatorname{tr}\left(\sum_{k \in \mathcal{S}} \int \frac{r_{k} d F^{\mathbf{R}_{k} \mathbf{P}_{k}}\left(r_{k}\right)}{1+\frac{r_{k}}{c_{k}} e_{k}(z)} \mathbf{T}_{k}-z \mathbf{I}_{N}\right)^{-1}
$$


where the $e_{i}$ 's verify

$$
e_{i}(z)=\frac{1}{N} \operatorname{tr} \mathbf{T}_{i}\left(\sum_{k \in \mathcal{S}} \int \frac{r_{k} d F^{\mathbf{R}_{k} \mathbf{P}_{k}}\left(r_{k}\right)}{1+\frac{r_{k}}{c_{k}} e_{k}(z)} \mathbf{T}_{k}-z \mathbf{I}_{N}\right)^{-1}
$$

From these equations, the complete MAC capacity region can be described. Denoting $\mathbf{R}_{k}=\mathbf{U}_{k} \boldsymbol{\Lambda}_{k} \mathbf{U}_{k}^{\mathrm{H}}$ the spectral decomposition of $\mathbf{R}_{k}$, we consider the power allocation strategy which consists in aligning the signal covariance matrix $\mathbf{P}_{k}$ to the eigenvectors of $\mathbf{R}_{k}$, i.e. $\mathbf{P}_{k}=\mathbf{U}_{k} \mathbf{Q}_{k} \mathbf{U}_{k}^{\mathrm{H}}$ for some diagonal matrix $\mathbf{Q}_{k}$. Therefore $\mathbf{P}_{i}$ in Equation (10) can be replaced by $\mathbf{U}_{i} \mathbf{Q}_{i} \mathbf{U}_{i}^{\mathrm{H}}$. The trace constraint in (10) is equivalent to $\operatorname{tr}\left(\mathbf{Q}_{i}\right) \leq P_{i}, i=1, \ldots, K$.

Remark 1 This power allocation strategy is only proven optimal if the $\mathbf{R}_{k}$ 's, $k \in\{1, \ldots, K\}$, are all equal [10].

Denoting $\mathbf{Q}_{k}=\operatorname{diag}\left(\mathbf{q}_{k}\right)=\operatorname{diag}\left(q_{k, 1}, \ldots, q_{k, n_{k}}\right)$ and $\Lambda_{k}=\operatorname{diag}\left(\lambda_{k, 1}, \ldots, \lambda_{k, n_{k}}\right)$, the Stieltjes transform of Equation (12) can be rewritten

$m_{\mathcal{S}}^{o}(z)=\frac{1}{N} \operatorname{tr}\left(\sum_{k \in \mathcal{S}} \frac{1}{n_{k}} \sum_{n=1}^{n_{k}} \frac{\lambda_{k, n} q_{k, n}}{1+\lambda_{k, n} q_{k, n} \frac{e_{k}(z)}{c_{k}}} \mathbf{T}_{k}-z \mathbf{I}_{N}\right)^{-1}$

To derive the MAC rate region polytope, for every set $\mathcal{S} \subset\{1, \ldots, K\}$, the sum rate (11) must be maximized, under the $|\mathcal{S}|$ constraints $\operatorname{tr}\left(\mathbf{Q}_{k}\right) \leq P_{k}$ and the $\sum_{i=1}^{|\mathcal{S}|} n_{i}$ constraints $q_{k, i} \geq 0$, for all $k \in \mathcal{S}$. This can be solved by classical convex optimization tools. Indeed, we have the following,

Proposition 3 The right-hand side of Equation (11) is a convex function of the $q_{k n}$ 's, $k \in \mathcal{S}, n \in\left\{1, \ldots, n_{k}\right\}$.

The proof and optimization details are developed in [5].

\subsection{Broadcast Channels}

The capacity region of the broadcast multi-antenna channel has been recently shown [8] to be achieved by the dirty-paper coding (DPC) algorithm. This region $\mathrm{C}_{\mathrm{BC}}(P ; \mathbf{H})$, for a transmit power constraint $P$ over the compound channel $\mathbf{H}$, is shown by duality arguments to be the set [7]

$$
\mathcal{C}_{\mathrm{BC}}(P ; \mathbf{H})=\bigcup_{\sum_{k=1}^{K} P_{k} \leq P} \mathcal{C}_{\mathrm{MAC}}\left(P_{1}, \ldots, P_{K} ; \mathbf{H}^{\mathrm{H}}\right)
$$

which is immediately obtained from Equation (10).

\section{SIMULATION AND RESULTS}

In this section, we provide simulation results in the context of a two-user broadcast channel, with $N=8$ transmit antennas and $n_{1}=n_{2}=4$ receive antennas, all placed in linear arrays. The space $d_{\mathrm{T}}$ between consecutive transmit antennas is such

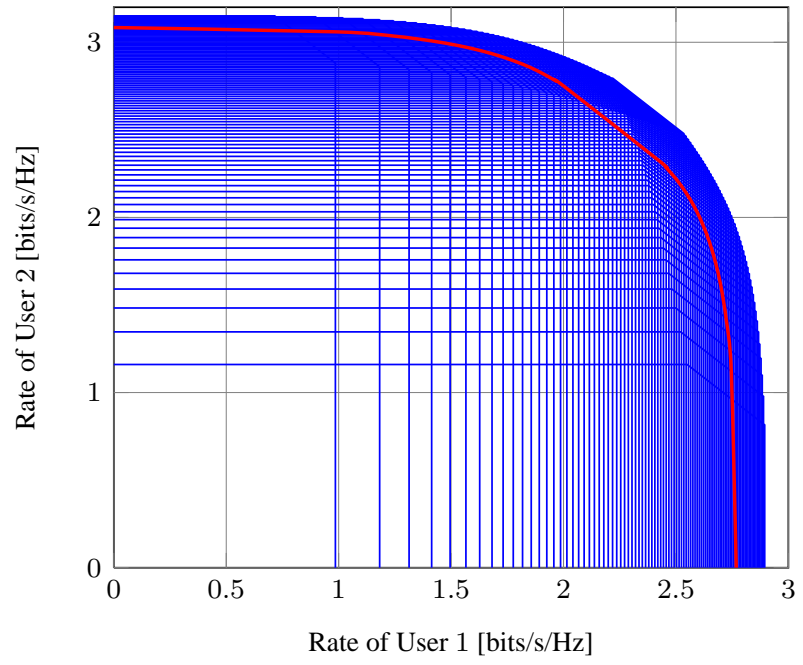

Fig. 1. $\mathcal{C}_{\mathrm{BC}}$ for $K=2$ users, $N=8, n_{1}=n_{2}=4$, $\mathrm{SNR}=$ $20 \mathrm{~dB}$, random transmit-receive solid angle of aperture $\pi / 2$, $d_{\mathrm{T}} / \lambda=10, d_{\mathrm{R}} / \lambda=1 / 4$. In thick line, $\mathrm{E}\left[\mathbf{s s}^{\mathrm{H}}\right]=\mathbf{I}_{N}$.

that $d_{\mathrm{T}} / \lambda=10$, with $\lambda$ the transmit signal wavelength; between adjacent receive antennas, the distance $d_{\mathrm{R}}$ is the same for users 1 and 2 and such that $d_{\mathrm{R}} / \lambda=1 / 4$. The variance profile is generated from Jakes' model, with privileged directions of signal departure and arrival (those directions form the solid angles under which the useful signals are transmitted/received). For instance, the entry $(a, b)$ of the transmit correlation matrix $\mathbf{T}_{1}$ is

$$
T_{1_{a b}}=\int_{\theta_{\min }^{\left(\mathbf{T}_{1}\right)}}^{\theta_{\max }^{\left(\mathbf{T}_{1}\right)}} \exp \left(2 \pi \cdot i \cdot|a-b| \frac{d_{\mathrm{T}}}{\lambda} \cos (\theta)\right) d \theta
$$

where, in our case, with obvious notations, $\theta_{\min }^{\left(\mathbf{R}_{1}\right)}=0, \theta_{\max }^{\left(\mathbf{R}_{1}\right)}=$ $\pi / 2, \theta_{\min }^{\left(\mathbf{R}_{2}\right)}=\pi / 3, \theta_{\max }^{\left(\mathbf{R}_{2}\right)}=5 \pi / 6, \theta_{\min }^{\left(\mathbf{T}_{1}\right)}=2 \pi / 3, \theta_{\max }^{\left(\mathbf{T}_{1}\right)}=$ $-5 \pi / 6, \theta_{\min }^{\left(\mathbf{T}_{2}\right)}=\pi, \theta_{\max }^{\left(\mathbf{T}_{2}\right)}=-\pi / 2$.

In Figure 1, we consider a $20 \mathrm{~dB}$ SNR and compare the rate region obtained from convex optimization to the equal power allocation strategy. We observe that, in spite of the strong correlation leading to very low eigenvalues in $\mathbf{R}_{1}$ and $\mathbf{R}_{2}$, little is gained by power allocation. Quite to the contrary, in Figure 2, we consider a $-5 \mathrm{~dB}$ SNR, and observe a substantial gain in sum rate from our suboptimal scheme ${ }^{2}$ compared to the equal power allocation strategy.

Figures 1 and 2 confirm and quantify the result given in [11], which mainly states that for low SNR the sum-rate capacity of broadcast channels is asymptotically equal to the sum-rate capacity of the time-division multiple access (TDMA) strategy; therefore, for low SNR, no significant sum-rate gain is obtained by the computationally involved, yet optimal, dirtypaper coding compared to the simpler TDMA method, which merely consists in a time-division user scheduling.

\footnotetext{
${ }^{2}$ we remind that the optimality of this scheme is not proven to this day.
} 


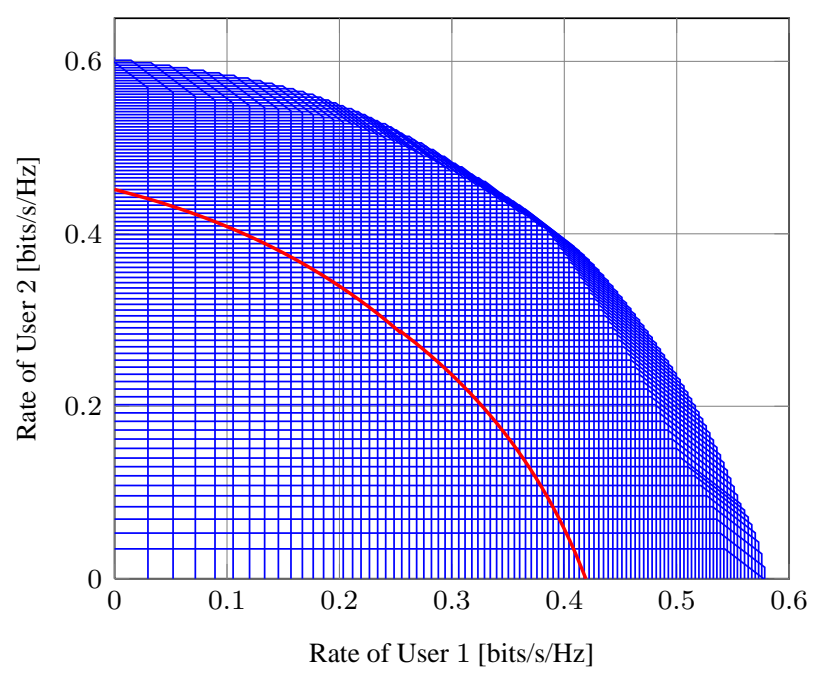

Fig. 2. $\mathcal{C}_{\mathrm{BC}}$ for $K=2$ users, $N=8, n_{1}=n_{2}=4$, $\mathrm{SNR}=$ $-5 \mathrm{~dB}$, random transmit-receive solid angle of aperture $\pi / 2$, $d_{\mathrm{T}} / \lambda=10, d_{\mathrm{R}} / \lambda=1 / 4$. In thick line, $\mathrm{E}\left[\mathbf{s s}^{\mathrm{H}}\right]=\mathbf{I}_{N}$.

In Figure 3, a comparison is made between the theoretical and simulated rate regions for a $20 \mathrm{~dB}$ SNR. The latter is obtained from 1,000 averaged Monte Carlo simulations for every transmit power pair $\left(P_{1}, P_{2}\right)$. We observe an almost perfect fit, even for these low $N=8, n_{1}=n_{2}=4$ numbers of transmit and receive antennas.

\section{CONCLUSION}

In this paper, we provided a compact expression for the rate region of multiple access and broadcast channels when the propagation channels are modelled as Gaussian with separable variance profiles. This result allows us to derive asymptotic capacity expressions of large antenna transmissions, independently of the specific channel realization but only based on the different transmit and receive correlation figures. While our results are only true for large numbers of transmit and receive antennas, we observe that the capacity results are good approximates even for small numbers of antennas.

\section{REFERENCES}

[1] E. Telatar, "Capacity of multi-antenna Gaussian channels," European transactions on telecommunications, vol. 10, no. 6, pp. 585-595, 1999.

[2] A. M. Tulino, "Impact of Antenna Correlation on the Capacity of Multiantenna Channels," IEEE Trans. on Information Theory, vol. 51, no. 7, 2005.

[3] A. Moustakas, S. Simon, A. Sengupta, "MIMO Capacity Through Correlated Channels in the Presence of Cor-

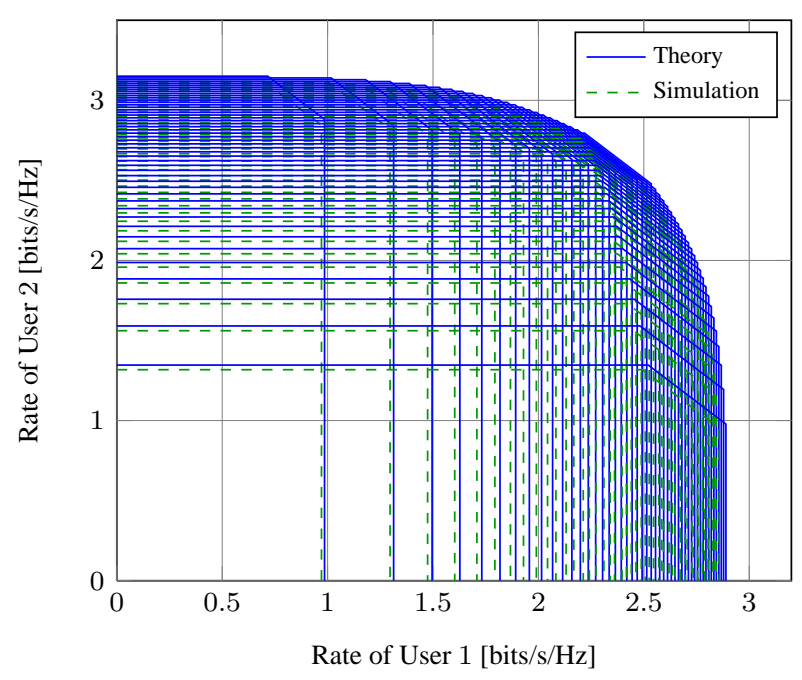

Fig. 3. $\mathcal{C}_{\mathrm{BC}}$ for $K=2$ users, theory against simulation, $N=$ $8, n_{1}=n_{2}=4, \mathrm{SNR}=20 \mathrm{~dB}$, random transmit-receive solid angle of aperture $\pi / 2, d_{\mathrm{T}} / \lambda=10, d_{\mathrm{R}} / \lambda=1 / 4$.

related Interferers and Noise: A (Not So) Large $N$ Analysis," IEEE Trans. on IT, vol. 49, no. 10, 2003.

[4] A. Soysal and S. Ulukus, "Optimum Power Allocation for Single-User MIMO and Multi-User MIMO-MAC with Partial CSI," IEEE Journal on Selected Areas in Communications, vol. 25, no. 7, 2007.

[5] R. Couillet, M. Debbah, J. Silverstein, "A deterministic equivalent approach for the capacity analysis of multiuser MIMO channels,"submitted to IEEE Trans. on Information Theory.

[6] S. Verdù, "Multiple-access channels with memory with and without frame synchronism," IEEE Trans. on Information Theory, vol. 35, pp. 605-619, 1989.

[7] S. Vishwanath, N. Jindal and A. Goldsmith, "Duality, Achievable Rates, and Sum-Rate Capacity of Gaussian MIMO Broadcast Channels," IEEE Trans. on Information Theory, vol. 49, no. 10, 2003.

[8] H. Weingarten, Y. Steinberg and S. Shamai, "The Capacity Region of the Gaussian Multiple-Input MultipleOutput Broadcast Channel," IEEE Trans. on Information Theory, vol. 52, no. 9, pp. 3936-3964, 2006.

[9] A. Tulino and S. Verdú, "Random Matrix Theory and Wireless Communications,” Now Publishers, 2004.

[10] A. Soysal and S. Ulukus "Optimality of Beamforming in Fading MIMO MAC Channels," to be published.

[11] N. Jindal and A. Goldsmith, "Dirty-paper coding versus TDMA for MIMO broadcast channels," IEEE Trans. on Information Theory, vol. 51, no. 5, pp. 1783-1794, 2005. 